\title{
Localized plateau beam resulting from strong nonlocal coupling in a cavity filled by metamaterials and liquid-crystal cells
}

\author{
M. Tlidi, ${ }^{1}$ C. Fernandez-Oto, ${ }^{1}$ M. G. Clerc, ${ }^{2}$ D. Escaff, ${ }^{3}$ and P. Kockaert ${ }^{4}$ \\ ${ }^{1}$ Faculté des Sciences, Université libre de Bruxelles (U.L.B.), C.P. 231, Campus Plaine, B-1050 Bruxelles, Belgium \\ ${ }^{2}$ Departamento de Física, Universidad de Chile, Blanco Encalada 2008, Santiago, Chile \\ ${ }^{3}$ Complex Systems Group, Facultad de Ingeniería y Ciencias Aplicadas, Universidad de los Andes, \\ Av. Mon. Alvaro del Portillo 12.455, Santiago, Chile \\ ${ }^{4}$ OPERA-photonique, Université libre de Bruxelles (U.L.B.), CP 194/5, 50, Av. F.D. Roosevelt, B-1050 Bruxelles, Belgium
}

(Received 28 May 2015; published 17 November 2015)

\begin{abstract}
We investigate the formation of a localized plateau beam in the transverse section of a nonlinear optical ring cavity filled with a metamaterial and a nonlocal medium such as a nematic liquid crystal. We show that, far from the modulational instability regime, localized structures with a varying width may be stable in one and two-dimensional settings. The mechanism of stabilization is related with strong nonlocal coupling mediated by a Lorentzian type of kernel. We show that there exists stable bright and dark localized structures. A reduction of Lugiato-Lefever equation in the regime close to the nascent bistability allows us to analytically derive a simple formula for the width of localized structures in one-dimensional systems. Direct numerical simulations of the dynamical model agree with the analytical predictions.
\end{abstract}

DOI: 10.1103/PhysRevA.92.053838

PACS number(s): 42.65.Tg, 42.70.-a, 05.45.-a, 42.65.-k

\section{INTRODUCTION}

The emergence of localized structures (LS's) in out-ofequilibrium systems has witnessed tremendous progress in the last two decades, allowing for the design of photonic devices for all-optical control of light, optical storage, and information processing (see recent overviews on this issue in Refs. [1-5]). These localized structures usually appear as the result of interactions between optical nonlinearity and other phenomena such as dispersion and diffraction. It is generally sufficient to consider that the nonlinear response is local, i.e., that the material response at a certain point depends only on the value of the electromagnetic field at that point. However, many materials exhibit spatial nonlocality, i.e., the refractive index at a certain point depends not only on the value of the field at that point, but also on the field in the region surrounding this point. The material response is therefore calculated by means of a spatial convolution between the excitation beam and a kernel called the "influence function," accounting for the nonlocality.

We classify the kernel functions into two types: weak [6-10] and strong [11-14]. If the kernel function decays asymptotically infinity slower (faster) than an exponential function, the nonlocal coupling is said to be strong (weak) [14]. However, our definition of strong nonlocality pertains only to the material properties, it differs from the usual one [15] that takes into account both the material response and the beam diameter, stating that the nonlocal coupling is strong if the width of the kernel functions is large in comparison with the beam diameter. Several experimental measurements of a strong nonlocal response of the material with a Lorentzian or a generalized Lorentzian shape have been carried out in nematic liquid-crystal (NLC) cells $[16,17]$. The NLC response was best fit with a pseudo-Lorentzian function, which corresponds also to a strong nonlocal coupling. Experimental reconstruction of strong nonlocal coupling has been performed in photorefractive materials [18].

In a different subfield of optics, left-handed materials (LHMs) are metamaterials that present a negative permittivity and permeability $[19,20]$. They were first demonstrated at microwave frequencies [21,22] and soon after in the optical domain $[23,24]$. Metamaterials are shown to exhibit novel electromagnetic phenomena, such as subwavelength imaging $[25,26]$ or negative diffraction, and can be used in nonlinear optical devices [3,27-30]. In particular, the formation of both conservative and dissipative solitons in a Kerr resonator containing a LHM was studied in Refs. [29,31-37].

In this paper, we consider a ring resonator filled with two materials having indices of refraction of opposite signs (see Fig. 1). The first material exhibits a strong nonlocal coupling mediated by a Lorentzian, while the second material consists of a linear material with a negative-diffraction coefficient. We focus on the bistable regime far from any modulational or Turing type of instabilities. We analyze the interaction of fronts connecting the two stable homogeneous steady states and we investigate the impact of strong nonlocal coupling on front propagation in the system. A front is a heteroclinic connection between homogeneous steady states [38,39]. Generally, the fronts are propagating, which means that they are moving and interacting.

When the nonlocal coupling is weak, the interaction of fronts is usually described by the behavior of the tail of one front around the core of the other front [6,8]. However, for strong nonlocal coupling, the interaction is controlled by the whole influence function and not only by the asymptotic behavior of the front tails. Recently, we reported that a strong nonlocal interaction is responsible for a new mechanism to stabilize a single localized structure [11-13]. We showed that strong nonlocal coupling drastically alters the spacetime behavior of spatially extended systems by affecting the asymptotic behavior of a single front and by modifying the law governing front interactions. We also demonstrated the occurrence of stable localized structures with varying plateau size. In the current context, the mechanism of stabilization is attributed to the combined influence of the strong nonlocal coupling in the nematic liquid crystal, negative diffraction in 


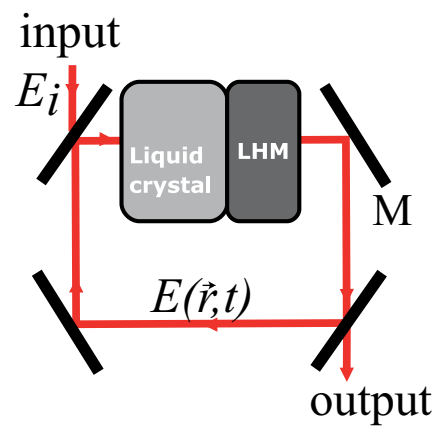

FIG. 1. (Color online) Ring resonator filled with a left-handed material (LHM) and a nonlocal medium such a liquid crystal. $M$ accounts for the mirrors.

the metamaterial, and bistability induced by the presence of an optical resonator.

The paper is organized as follows: After introducing the model (Sec. II), we perform numerical simulations of the full model (Sec. III). A reduction of the equation in the nascent bistability regime, which allows us to analytically describe the interaction of the fronts, is presented in Sec. IV. We conclude in Sec. V.

\section{LUGIATO-LEFEVER MODEL WITH NONLOCAL COUPLING}

The ring resonator filled with a material presenting a nonlocal nonlinearity (NLNL) and a negative-diffraction (ND) material is described by the well-known Lugiato-Lefever (LL) model [40], in which we incorporate the strong nonlocal effect. This model equation is valid under the following approximations: (i) at the interface separating the NLNL and the ND materials, the reflection is assumed to be negligible, i.e., they are impedance matched; (ii) the cavity possesses a high Fresnel number, i.e., it is a large-aspect-ratio system and we assume that the cavity is much shorter than the diffraction and the nonlinearity spatial scales; (iii) for the sake of simplicity, we assume single-longitudinal-mode operation. Under these assumptions the spacetime evolution of the intracavity field is described by the following partial differential equation:

$$
\begin{aligned}
\partial_{t} E= & E_{i}-(1+i \theta) E+i|E|^{2} E+i D \nabla^{2} E \\
& -i \gamma \int_{\Omega} E\left(\mathbf{r}+\mathbf{r}^{\prime}, t\right) K\left(\mathbf{r}^{\prime}\right) d \mathbf{r}^{\prime} .
\end{aligned}
$$

The normalized slowly varying complex envelope of the electric field is $E$. The input field amplitude $E_{i}$ is real and constant. The detuning parameter is given by $\theta$. The diffraction coefficient is $D$, and $\Omega$ is the domain under study. In the absence of nonlocal coupling in the liquid-crystal cell, i.e., $\gamma=0$, we recover the well-known Lugiato-Lefever model [40]. The inclusion of a LHM slice allows us to explore the parameter regime where diffraction is negative [31]. From a practical point of view, negative diffraction could be achieved by using a self-imaging configuration [41-43]. In the absence of nonlocal interaction, the stabilization of localized solutions far from any pattern-forming instability has been realized thanks to the combined action of negative diffraction and an inhomogeneous-pumping laser beam [43]. The laser cavity can be also considered as an inhomogeneous cavity in the work by Zhang $[44,45]$. Finally, the nonlocal coupling is modeled by the function $K\left(\mathbf{r}^{\prime}\right)$ :

$$
K\left(\mathbf{r}^{\prime}\right)=\delta\left(\mathbf{r}^{\prime}\right)-f_{\sigma}\left(\mathbf{r}^{\prime}\right)
$$

where $\delta\left(\mathbf{r}^{\prime}\right)$ is the delta distribution and the kernel function $f_{\sigma}\left(\mathbf{r}^{\prime}\right)$ is defined by

$$
f_{\sigma}(\mathbf{r})=\frac{N_{n}}{1+(|\mathbf{r}| / \sigma)^{n}}
$$

The parameter $\gamma$ measures the intensity of the nonlocal interaction, $\sigma$ represents the characteristic length of the nonlocal interaction, and $N_{n}$ is a normalization constant. Such a nonlocal response can be realized in a liquid-crystal cell. In particular, experimental measurements [16] show that the nonlocal variation of the refractive index in cells filled with the commercially available E7 liquid crystal is well fit with a Lorentzian. In this experiment, the thickness of the cell was varied over the range 18 to $73 \mu \mathrm{m}$. Another group of researchers reported slightly different results obtained by using pulsed beams [17]. The nonlocal response of the material was deduced from the interaction of soliton beams in a liquid-crystal cell. This response was best fit with a pseudo-Lorentzian function, which corresponds also to a strong nonlocal coupling, as defined above. Experimental evidence of strong nonlocal coupling has also been performed in photorefractive materials [18], where the strong nonlocal coupling originates from thermal effects.

The homogeneous steady states of Eq. (1) are solutions of $E_{i}=\left[1+i\left(\theta-\left|E_{s}\right|^{2}\right)\right] E_{s}$. The response curve giving the intracavity intensity $\left|E_{s}\right|^{2}$ as a function of the input intensity $\left|E_{i}\right|^{2}$ is monostable for $\theta<\sqrt{3}$ and exhibits a bistable behavior when the detuning $\theta>\sqrt{3}$ (see Fig. 2).

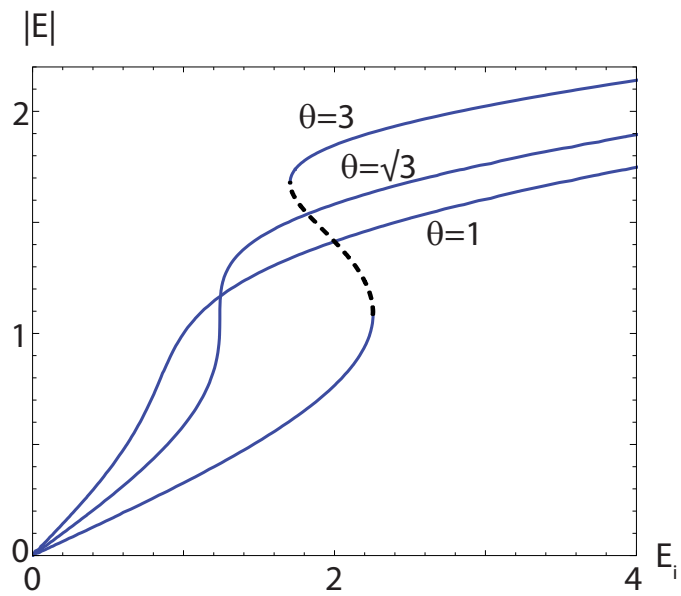

FIG. 2. (Color online) Homogeneous steady-state solutions of Eq. (1) for different values of the detuning parameter $\theta$. The continuous and dashed curves indicate stable and unstable states, respectively. 


\section{LOCALIZED PLATEAU BEAM: NUMERICAL SIMULATIONS}

Our numerical simulations are focused on the parameter range corresponding to bistability of the homogeneous steady-state solutions, i.e., $\theta>\sqrt{3}$ and we consider a regime where these states are modulationally stable far from any modulational or Turing instability. The latter condition is achieved when the effective diffraction of the cavity is negative $(D<0)$.

Numerical simulations generate stable one-dimensional localized structures, as shown in Fig. 3. From these plots, we can see that the width of the localized structures obtained vary strongly with the injected beam intensity. In addition these structures posses a plateau and the background exhibits spatial damped oscillations. The stabilization mechanism is robust in two dimensional setting. An example of two-dimensional (2D) localized structures with a varying-size plateau is shown in Fig. 4. As one-dimensional setting, these structures exhibit damped oscillations in the profile of the intracavity intensity. The circular flat area varies strongly with a small change of the injected beam intensity, as shown in Fig. 4.

Localized structures have been predicted in onedimensional (1D) bistable optical systems with a Gaussian beam [46-48]. These solutions were interpreted as switching waves connecting two stable branches of the homogeneous response curve. It was shown later that LS's do not require
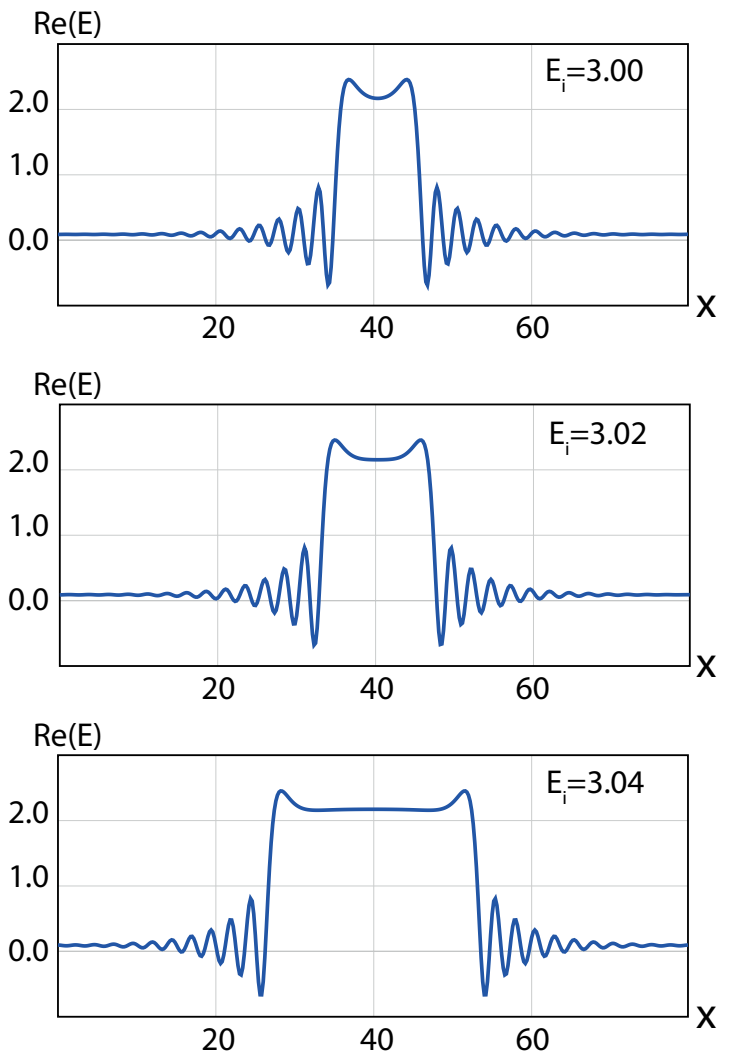

FIG. 3. (Color online) Localized plateau beam in one transverse dimension. Three different structures in one dimension, for three different values of injection $E_{i}$. The parameters are $\theta=6.0, D=$ $-1.0, \gamma=1.0, \sigma=0.7$, and $n=2.0$.
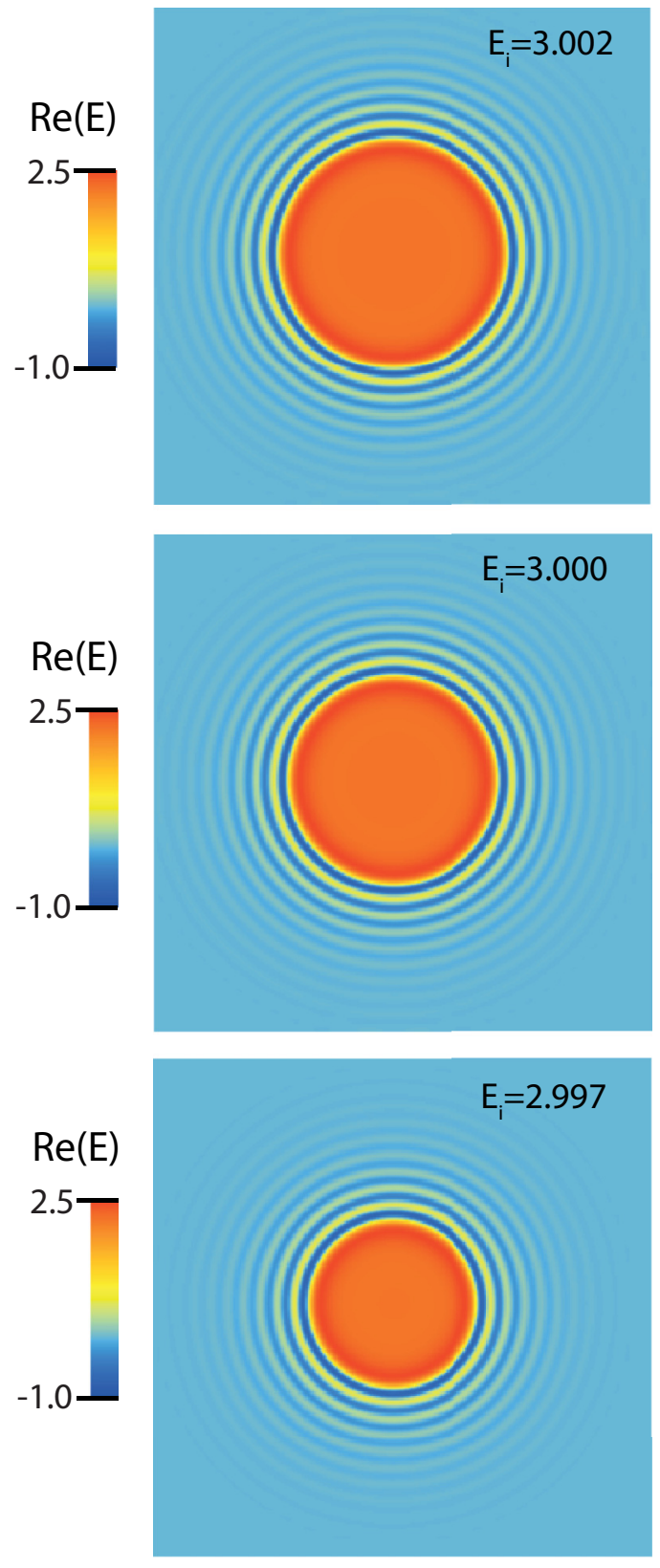

FIG. 4. (Color online) Localized plateau beam in two dimensions. Three different structures in two dimensions, for three different values of injection $E_{i}$. The parameters are $\theta=5.93, D=-1.0$, $\gamma=1.0, \sigma=0.4$, and $n=2.2$.

a commutation process between distinct uniform stationary states [49,50]. Two-dimensional LS's can be stable in the monostable regime where a single homogeneous steady state coexists with periodic structures such as hexagons and stripes [49-51]. In bistable regime, the physical systems exhibit the existence of a hysteresis loop. Within it, there generally exists a so-called pinning range of parameter values for which stable localized structures, connecting the uniform and the periodic solutions, can be found. The existence of a pinning range was predicted by Pomeau [52] and observed experimentally in a liquid-crystal light valve with optical feedback [53]. The formation of LS's in the Turing regime is common in a variety of systems such as reaction-diffusion 
(a)

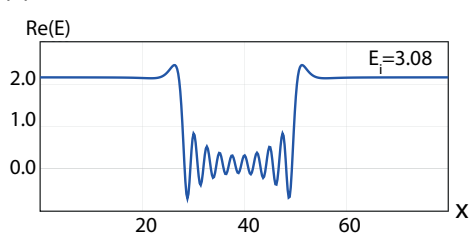

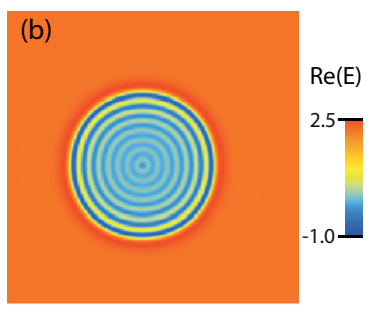

FIG. 5. (Color online) Dark LS's in one and two dimensions. In panel (a) the parameters are $\theta=6.0, D=-1.0, \gamma=1.0, \sigma=0.7$, $n=2.0$, and $E_{i}=3.08$. In panel (b) the parameters are $\theta=5.93$, $D=-1.0, \gamma=1.0, \sigma=0.4, n=2.2$, and $E_{i}=3.034$.

[54-56], hydrodynamics [57-63], photorefractives [64], Ginzburg-Landau equation [65,66], liquid crystals [51,6770], and semiconductors [71-73]. The bifurcation that leads to the formation of LS's is referred to as the homoclinic snaking phenomenon [74-80]. The relative stability of 1D and 2D clusters of closely packed localized peaks of the Swift-Hohenberg equation has been analyzed [81].

The localized plateau beams reported here are obtained in a regime devoid of any pattern-forming type of instability. They differ from the above-mentioned LS in three ways: First, a localized plateau beam cannot be stable in the monostable regime. Bistability is the prerequisite condition for their stabilization. Second, localized structures found in the subcritical Turing regime have a fixed width. Their width does not significantly change as a function of the injected beam intensity. Their width is determined by the most unstable wavelength. However, in our case the width of the LSs vary strongly with the injected beam intensity. Third, our LSs connect two homogeneous steady states while the LSs found in the Turing regime connect one homogeneous steady to a periodic pattern.

In the absence of strong nonlocal coupling $(\gamma=0)$, the model Eq. (1) does not admit stable bright localized structures with varying plateau size. The inclusion of strong nonlocal coupling allows for the stabilization of these localized states in one and two transverse directions, as shows in Figs. 3 and 4. In addition, dark localized structures may be stabilized by the fact that the lower uniform exhibits damped oscillations near to the front core. In Fig. 5 we can see the combination of both effects, strong nonlocal coupling and damped oscillations, stabilizing dark localized structures in one and two dimensions, as shown in Figs. 5(a) and 5(b). The damped oscillations appear for large detuning parameters. However, close to nascent optical bistability $(\theta-\sqrt{3} \ll 1)$, numerical simulations of the model Eq. (1) show that both dark and bright localized structures are devoid of damped oscillations (see Fig. 6).

In the next section we perform a perturbative calculation near the onset of bistability, which allows us to describe analytically the formation of localized structures with varying plateau size in one dimension.

\section{LOCALIZED PLATEAU BEAMS: ANALYTICAL ANALYSIS}

The analytical investigation of fronts dynamics connecting two-homogeneous steady states in the framework of Eq. (1) is a
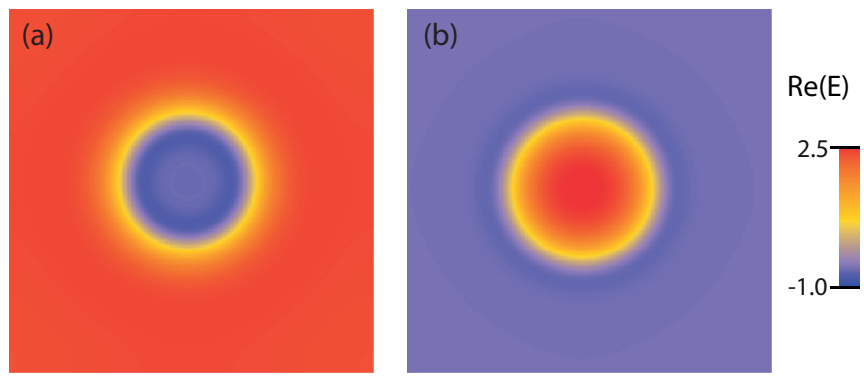

FIG. 6. (Color online) (a) Dark and (b) bright localized structures in two dimensions obtained by numerical simulations of Eq. (1). The parameters are $\theta=1.8, D=-1.0, \gamma=0.3, \sigma=0.4$, and $n=2.2$. The injection-field amplitude is (a) $E_{i}=1.278$ and (b) $E_{i}=1.275$.

complex problem. In this section we reduce Eq. (1) to a simple bistable model with nonlocal interaction. This reduction is valid close to the onset of optical bistability. At the critical point where the output versus input characteristics have an infinite slope, the critical detuning is $\theta=\theta_{c} \equiv \sqrt{3}$. In order to study the front dynamics, we explore the vicinity of the critical point associated with bistability. At the critical point, the coordinate of the intracavity are $E_{c}=u_{c}+i v_{c}$ with $u_{c}=3^{1 / 4} / \sqrt{2}$ and $v_{c}=-1 /\left(3^{1 / 4} \sqrt{2}\right)$, and the injected field amplitude is $E_{i c}=$ $2 \sqrt{2} / 3^{3 / 4}$. To derive a simple bistable model with nonlocal interaction, we introduce a small parameter $\epsilon$ which measures the distance from the critical point as $\theta=\theta_{c}+\theta_{0} \epsilon^{2}$. Then we decompose the field into its real and imaginary parts as $E=u+i v$ and introduce the excess variables $U$; and $V$ as $u=u_{c}+U$ and $v=v_{c}+V$. We next expand the $U, V$, and $E_{i}$ in power series of $\epsilon$ as

$$
\begin{aligned}
U & =\epsilon \sqrt{3 \theta_{0}} C / 2-\epsilon^{2} \theta_{0}\left[3^{3 / 4} C^{2} / \sqrt{2}-\sqrt{3} u_{c} / 2\right]+\cdots, \\
V & =\epsilon \sqrt{\theta_{0}} C / 2+\cdots, \\
E_{i} & =E_{c}-\epsilon^{2} \theta_{0} v_{c}+\epsilon^{3} \eta \theta_{0}^{3 / 2} / 2+\cdots,
\end{aligned}
$$

where $C\left(x_{0}, T\right)$ is an order parameter that describes the optical instability, and $T \equiv \epsilon^{2} \theta_{0} / \sqrt{3}$, and $x_{0} \equiv \epsilon x \sqrt{-\theta_{0} / D}$ rescale time and space, respectively. The intensity of the nonlocal coupling scale as $\gamma \equiv \theta_{0} \epsilon^{2} \gamma_{0}$. The application of the solvability condition at third order in $\epsilon$ leads to a simple bistable model with a nonlocal coupling:

$$
\begin{aligned}
\partial_{T} C= & \eta+C-C^{3}+\partial_{x_{0} x_{0}} C \\
& +\gamma_{0} \int_{-\infty}^{\infty} C\left(x_{0}+x_{0}^{\prime}, t\right) K\left(x_{0}^{\prime}\right) d x_{0}^{\prime} .
\end{aligned}
$$

In the absence of the nonlocal coupling, i.e., $\gamma_{0}=0$, we recover a bistable model [43]. In the expansion of the injected field appears an extra parameter $\eta$, which account for the deviation to the Maxwell point. The Maxwell point is obtained when the front velocity vanishes. This point corresponds to $\eta=0$.

In what follows we derive an equation for the time evolution of the width of localized structures. Then we seek for the stationary width as a function of the system parameters. For this purpose we restrict our analysis to the case of one transverse dimension. The two-dimensional case is far beyond the scope of the present paper. Close to the Maxwell point, one can consider the following ansatz for 
the localized structure: $C\left(x_{0}, T\right)=\tanh \left\{\left[x_{0}+\Delta_{0}(T)\right] / \sqrt{2}\right\}-$ $\tanh \left\{\left[x_{0}-\Delta_{0}(T)\right] / \sqrt{2}\right\}-1+\delta C$, where $2 \Delta_{0}$ stands for the width of the localized structure and $\delta C$ accounts for small corrections. To get the front dynamic, we introduce the above ansatz for $C$ in Eq. (4), linearize in $\delta C$, and imposing the solvability condition. We obtain for $n=2$,

$$
\partial_{T} \Delta_{0}=\frac{3 \eta}{\sqrt{2}}+\frac{3 \gamma_{0} \sigma \epsilon \sqrt{\theta_{0}}}{\sqrt{2} \pi \Delta_{0}}
$$

Then, the equilibrium size is give by

$$
\Delta_{0}^{e q}=\frac{-\gamma_{0} \sigma \epsilon \sqrt{\theta_{0}}}{\pi \eta} .
$$

In terms of the original parameters, the width of the structure is

$$
\Delta^{e q}=\frac{-\gamma \sigma \sqrt{\theta-\theta_{c}}}{\pi\left(E_{i}-E_{M}\right)},
$$

where $E_{M}$ is the value of the Maxwell point with the nonlocal coupling intensity $\gamma$ and the interaction range $\sigma$.

The formula Eq. (7) is valid close to the nascent optical bistability $(\theta-\sqrt{3} \ll 1)$. This prediction is in perfect agreement with numerical simulations. In order to check the robustness of the proposed stabilization mechanism, we perform the analysis far from the onset of bistability by fixing the detuning parameter to $\theta=6$. In this case, the Maxwell-point value $\left(E_{M}\right)$ depends strongly on the intensity of the nonlocal coupling $\gamma$ and the interaction range $\sigma$, as shown in Fig. 7. The comparison between the stationary size of localized structure obtained from the formula (7) fits quite well with the results of numerical simulations without any other adjustment parameter. The comparison is shown in Fig. 8. These plots show that our analytical investigation is still valid beyond the nascent optical bistability. The width of localized structure increases with the distance to the Maxwell point and with the intensity of the strong nonlocal coupling. At the Maxwell point, the width of localized structure diverges at the Maxwell point as shown in Fig. 8(a). The width of localized structure depends linearly on the nonlocal intensity $\gamma$, as shown in Fig. 8(b). Numerical results are in good agreement with the theoretical predictions.

The presence of well-pronounced oscillatory tails suggests a possibility to form robust bound complexes of two or several

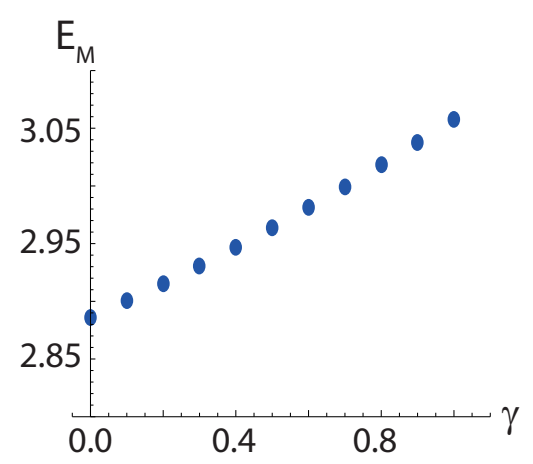

FIG. 7. (Color online) Numerical value for the Maxwell point as function of the nonlocal intensity $\gamma$. The parameters are $\theta=6.0$, $D=-1.0, \sigma=0.7$, and $n=2.0$. (a)

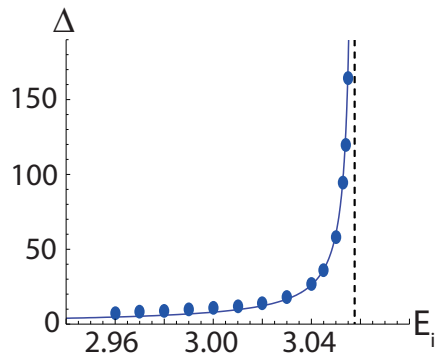

(b)

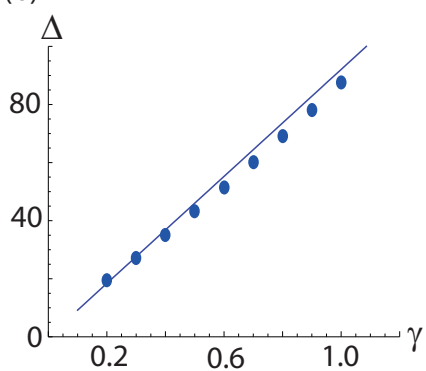

FIG. 8. (Color online) Theoretical and numerical size of localized plateau beam in one dimension. The solid line is the theoretical prediction from Eq. (7), the dots are the numerical results from Eq. (1), and the dashed line in panel (a) corresponds to the Maxwell point estimated numerically. In both graphics, the parameters are $\theta=6.0, D=-1.0, \sigma=0.7$, and $n=2.0$. In panel (a), the nonlocal intensity $\gamma=1.0$. In panel (b), the distance to the Maxwell point $E_{M}-E_{i}=0.005$.

localized plateau beams. An example of three localized beams bound together is shown in Fig. 9. When the distance between peaks decreases they start to interact via their oscillating, exponentially decaying tails. This interaction then leads to the formation of clusters [82-87].

To address the problem of experimental feasibility of localized plateau beams, we need to work with a stabilized, and slightly detuned, nonlinear and spatially extended optical cavity, with strong nonlocal coupling and with a layer of left-handed material. The nonlocal nonlinear material that we target is a liquid-crystal cell filled with a standard E7 liquid crystal and used in a geometry similar to that of Ref. [16]. Such a cell is strongly nonlocal, according to the experimental characterization reported in Ref. [16]. It presents also a high nonlinearity that allows us to perform the experiment with reasonable optical power. We need a pump beam that allows us to reach the input intensity at which the system switches to the higher homogeneous steady state. To keep a sufficient power margin, we work with a green $\mathrm{cw}$ laser delivering up to $15 \mathrm{~W}$ at a wavelength of $532 \mathrm{~nm}$. The control of the detuning is a key issue. For this purpose, we make use of an internally developed proportional-integral controller based on a microchip device. Once the stabilization setup is working with a beam polarized in one direction, the observation of the plateau solutions will be performed with a beam polarized orthogonally to the control

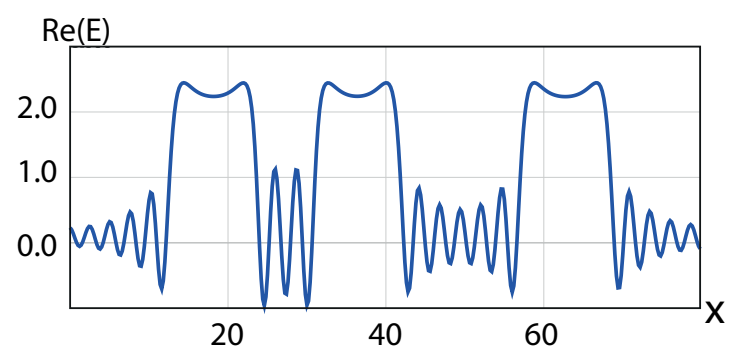

FIG. 9. (Color online) Interaction between three localized plateau beams in one transverse dimension. The parameters are $\theta=6.0, D=-1.0, \gamma=0.5, \sigma=0.7, E_{i}=2.94$, and $n=2.0$. 
beam as follows: First, for a given detuning, we record the input-output characteristic of the setup. For a sufficiently large detuning, we should observe bistability. The hysteresis curve allows us to determine the input power corresponding to the Maxwell point. Working at this power, and fixing the detuning, we induce the spatial structure by means of an additional seed that will be combined with the main beam outside the cavity. The pump beam will be split by a polarizing beam splitter followed on each arm by a different telescope, and by a half-wave plate on the second arm. The two arms will then be recombined on a nonpolarizing beam splitter in a Mach-Zehnder configuration. The relative width between the main beam and the seed beam will be fixed by the parameters of the two telescopes, while their relative intensity will be determined by the orientation of the wave plates. The output of the cavity will be recorded on a camera. This will allow to study the evolution of the plateau solution when varying the different control parameters (pump power, detuning, nonlinearity by application of a voltage on the liquid-crystal cell).

\section{CONCLUSIONS}

We considered a ring cavity filled by a combination of a nonlocal medium and a left-handed material. This cavity is driven by a coherent injected signal. We investigated the formation of localized structures in a bistable regime where both homogeneous steady states are modulationally stable. To avoid a modulational or Turing instability, we used a left-handed element that allows the system to operate in a negative-diffraction regime. The response of the left-handed material is considered to be linear, and the nonlinearity of the Kerr type originates from the nonlocal medium, such as nematic-liquid-crystal cells. We presented a mechanism of generation of stable localized structures based on strong nonlocal coupling mediated by a Lorentzian-like kernel. This type of nonlocal coupling has been reported experimentally in the case of nematic crystals cells $[16,17]$. This nonlocal coupling modifies the nature of the front interaction between two homogeneous steady states and allows for the stabilization of both bright and dark localized structures. These structures have varying plateau size. Without strong nonlocal coupling, localized structures are unstable. They either shrink or expand. An analytical expression of the front interaction law is provided in the regime close to the critical point associated with bistability. Close to this point we reduced the LL (1) to a simple bistable-model equation with nonlocal interaction. The variation of the width of localized structures was derived in a one-dimensional system within the framework of the reduced equation. This generic mechanism is robust in one and two spatial dimensions and could be applied to a large class of far-from-equilibrium systems with strong nonlocal coupling. Finally, we discuss the experimental feasibility of localized plateau beams.

\section{ACKNOWLEDGMENTS}

M.G.C. acknowledges the financial support of FONDECYT Project 1150507. D.E. acknowledges the financial support of FONDECYT Project No. 1140128. C.F.-O. acknowledges financial support of Becas Chile. M.T. received support from the Fonds National de la Recherche Scientifique (Belgium) and the Interuniversity Attraction Poles program Photonics@be of the Belgian Science Policy Office (BelSPO), under Grant No. IAP 7-35.
[1] H. Leblond and D. Mihalache, Phys. Rep. 523, 61 (2013).

[2] M. Tlidi, K. Staliunas, K. Panajotov, A. G. Vladimiorv, and M. Clerc, Philos. Trans. R. Soc., A 372, 20140101 (2014).

[3] M. Lapine, I. V. Shadrivov, and Y. S. Kivshar, Rev. Mod. Phys. 86, 1093 (2014).

[4] L. Lugiato, F. Prati, and M. Brambilla, Nonlinear Optical Systems (Cambridge University Press, United Kingdom, 2015).

[5] Localized States in Physics: Solitons and Patterns, edited by O. Descalzi, M. Clerc, S. Residori, and G. Assanto (Springer, Berlin, 2010).

[6] M. G. Clerc, D. Escaff, and V. M. Kenkre, Phys. Rev. E 72, 056217 (2005)

[7] L. Gelens, D. Gomila, G. Van der Sande, M. A. Matías, and P. Colet, Phys. Rev. Lett. 104, 154101 (2010).

[8] M. G. Clerc, D. Escaff, and V. M. Kenkre, Phys. Rev. E 82, 036210 (2010)

[9] P. Colet, M. A. Matías, L. Gelens, and D. Gomila, Phys. Rev. E 89, 012914 (2014).

[10] L. Gelens, M. A. Matías, D. Gomila, T. Dorissen, and P. Colet, Phys. Rev. E 89, 012915 (2014).

[11] C. Fernandez-Oto, M. G. Clerc, D. Escaff, and M. Tlidi, Phys. Rev. Lett. 110, 174101 (2013).

[12] C. Fernandez-Oto, M. Tlidi, D. Escaff, and M. G. Clerc, Philos. Trans. R. Soc., A 372, 20140009 (2014).
[13] D. Escaff, C. Fernandez-Oto, M. G. Clerc, and M. Tlidi, Phys. Rev. E 91, 022924 (2015).

[14] D. Escaff, Eur. Phys. J. D 62, 33 (2011).

[15] W. Krolikowski and O. Bang, Phys. Rev. E 63, 016610 (2000).

[16] X. Hutsebaut, C. Cambournac, M. Haelterman, J. Beeckman, and K. Neyts, J. Opt. Soc. Am. B 22, 1424 (2005); G. Agez, P. Glorieux, C. Szwaj, and E. Louvergneaux, Opt. Commun. 245, 243 (2005).

[17] J. F. Henninot, J. F. Blach, and M. Warenghem, J. Opt. A 9, 20 (2007).

[18] A. Minovich, D. N. Neshev, A. Dreischuh, W. Krolikowski, and Y. S. Kivshar, Opt. Lett. 32, 1599 (2007).

[19] V. G. Veselago, Sov. Phys. Usp. 10, 509 (1968).

[20] J. B. Pendry, Phys. Rev. Lett. 85, 3966 (2000).

[21] R. A. Shelby, D. R. Smith, and S. Schultz, Science 292, 77 (2001).

[22] K. Aydin, K. Guven, M. Kafesaki, L. Zhang, C. M. Soukoulis, and E. Ozbay, Opt. Lett. 29, 2623 (2004).

[23] C. Enkrich, M. Wegener, S. Linden, S. Burger, L. Zschiedrich, F. Schmidt, J. F. Zhou, T. Koschny, and C. M. Soukoulis, Phys. Rev. Lett. 95, 203901 (2005).

[24] T. F. Gundogdu, I. Tsiapa, A. Kostopoulos, G. Konstantinidis, N. Katsarakis, R. S. Penciu, M. Kafesaki, E. N. Economou, T. Koschny, and C. M. Soukoulis, Appl. Phys. Lett. 89, 084103 (2006). 
[25] D. R. Smith, J. B. Pendry, and M. C. K. Wiltshire, Science 305, 788 (2004).

[26] M. W. Feise, I. V. Shadrivov, and Y. S. Kivshar, Phys. Rev. E 71, 037602 (2005).

[27] A. A. Zharov, N. A. Zharova, I. V. Shadrivov, and Y. S. Kivshar, Appl. Phys. Lett. 87, 091104 (2005).

[28] N. Lazarides and G. P. Tsironis, Phys. Rev. E 71, 036614 (2005).

[29] G. D’Aguanno, N. Mattiucci, M. Scalora, and M. J. Bloemer, Phys. Rev. Lett. 93, 213902 (2004).

[30] M. Scalora, M. S. Syrchin, N. Akozbek, E. Y. Poliakov, G. D'Aguanno, N. Mattiucci, M. J. Bloemer, and A. M. Zheltikov, Phys. Rev. Lett. 95, 013902 (2005).

[31] P. Kockaert, P. Tassin, G. Van der Sande, I. Veretennicoff, and M. Tlidi, Phys. Rev. A 74, 033822 (2006).

[32] P. Tassin, G. Van der Sande, N. Veretenov, P. Kockaert, I. Veretennicoff, and M. Tlidi, Opt. Express 14, 9338 (2006).

[33] P. Tassin, L. Gelens, J. Danckaert, I. Veretennicoff, G. Van der Sande, P. Kockaert, and M. Tlidi, Chaos 17, 037116 (2007).

[34] L. Gelens, G. Van der Sande, P. Tassin, M. Tlidi, P. Kockaert, D. Gomila, I. Veretennicoff, and J. Danckaert, Phys. Rev. A 75, 063812 (2007).

[35] A. D. Boardman, N. King, R. C. Mitchell-Thomas, V. N. Malnev, and Y. G. Rapoport, Metamaterials 2, 145 (2008).

[36] A. B. Kozyrev, I. V. Shadrivov, and Y. S. Kivshar, Appl. Phys. Lett. 104, 084105 (2014).

[37] A. D. Boardman, K. L. Tsakmakidis, R. C. Mitchell-Thomas, N. J. King, Y. G. Rapoport, and O. Hess, Springer Ser. Mater. Sci. 200, 161 (2015).

[38] M. C. Cross and P. C. Hohenberg, Rev. Mod. Phys. 65, 851 (1993).

[39] W. van Saarloos, Phys. Rep. 386, 29 (2003).

[40] L. A. Lugiato and R. Lefever, Phys. Rev. Lett. 58, 2209 (1987).

[41] J. A. Arnaud, Appl. Opt. 8, 189 (1969).

[42] P. Kockaert, J. Opt. Soc. Am. B 26, 1994 (2009).

[43] V. Odent, M. Tlidi, M. G. Clerc, P. Glorieux, and E. Louvergneaux, Phys. Rev. A 90, 011806(R) (2014).

[44] H. Zhang, D. Y. Tang, L. M. Zhao, and X. Wu, Phys. Rev. B 80, 052302 (2009).

[45] H. Zhang, D. Y. Tang, L. Zhao, and X. Wu, Opt. Express 19, 3525 (2011).

[46] J. V. Moloney and H. M. Gibbs, Phys. Rev. Lett. 48, 1607 (1982).

[47] D. W. Mc Laughlin, J. V. Moloney, and A. C. Newell, Phys. Rev. Lett. 51, 75 (1983).

[48] N. Rosanov and G. Khodova, Opt. Spectrosc. 65, 1399 (1988).

[49] A. J. Scroggie et al., Chaos, Solitons Fractals 4, 1323 (1994).

[50] M. Tlidi, P. Mandel, and R. Lefever, Phys. Rev. Lett. 73, 640 (1994).

[51] U. Bortolozzo, M. G. Clerc, and S. Residori, New J. Phys. 11, 093037 (2009).

[52] Y. Pomeau, Physica D 23, 3 (1986).

[53] F. Haudin, R. G. Elias, R. G. Rojas, U. Bortolozzo, M. G. Clerc, and S. Residori, Phys. Rev. Lett. 103, 128003 (2009).

[54] S. Koga and Y. Kuramoto, Prog. Theor. Phys. 63, 106 (1980).

[55] P. B. Umbanhowar, F. Melo, and H. L. Swinney, Nature (London) 382, 793 (1996).

[56] M. G. Clerc, E. Tirapegui, and M. Trejo, Phys. Rev. Lett. 97, 176102 (2006).
[57] P. Kolodner, D. Bensimon, and C. M. Surko, Phys. Rev. Lett. 60, 1723 (1988).

[58] O. Thual and S. Fauve, J. Phys. (Paris) 49, 1829 (1988).

[59] W. Barten, M. Lücke, and M. Kamps, Phys. Rev. Lett. 66, 2621 (1991).

[60] M. Dennin, G. Ahlers, and D. S. Cannell, Science 272, 388 (1996).

[61] H. Riecke, Phys. Rev. Lett. 68, 301 (1992).

[62] H. Riecke and G. D. Granzow, Phys. Rev. Lett. 81, 333 (1998).

[63] C. Crawford and H. Riecke, Physica D 129, 83 (1999).

[64] A. A. Zozulya, M. Saffman, and D. Z. Anderson, Phys. Rev. Lett. 73, 818 (1994).

[65] B. A. Malomed and A. A. Nepomnyashchy, Phys. Rev. A 42, 6009 (1990).

[66] V. Skarka, N. B. Aleksic, M. Lekic, B. N. Aleksic, B. A. Malomed, D. Mihalache, and H. Leblond, Phys. Rev. A 90, 023845 (2014).

[67] P. L. Ramazza, S. Ducci, S. Boccaletti, and F. T. Arecchi, J. Opt. B: Quantum Semiclassical Opt. 2, 399 (2000).

[68] U. Bortolozzo, L. Pastur, P. L. Ramazza, M. Tlidi, and G. Kozyreff, Phys. Rev. Lett. 93, 253901 (2004).

[69] U. Bortolozzo, M. G. Clerc, C. Falcon, S. Residori, and R. Rojas, Phys. Rev. Lett. 96, 214501 (2006).

[70] V. Odent, M. Taki, and E. Louvergneaux, New J. Phys. 13, 113026 (2011).

[71] V. B. Taranenko, K. Staliunas, and C. O. Weiss, Phys. Rev. A 56, 1582 (1997).

[72] V. B. Taranenko, K. Staliunas, and C. O. Weiss, Phys. Rev. Lett. 81, 2236 (1998).

[73] E. Averlant, M. Tlidi, H. Thienpont, T. Ackemann, and K. Panajotov, Opt. Express 22, 762 (2014).

[74] A. R. Campneys, Physica D 112, 158 (1998).

[75] Y. Nishiura and D. Ueyama, Physica D 130, 73 (1999).

[76] P. Coullet, C. Riera, and C. Tresser, Phys. Rev. Lett. 84, 3069 (2000).

[77] M. G. Clerc, C. Falcon, and E. Tirapegui, Phys. Rev. Lett. 94, 148302 (2005).

[78] M. Tlidi and L. Gelens, Opt. Lett. 35, 306 (2010).

[79] M. Tlidi, P. Kockaert, and L. Gelens, Phys. Rev. A 84, 013807 (2011).

[80] F. Haudin, R. G. Rojas, U. Bortolozzo, S. Residori, and M. G. Clerc, Phys. Rev. Lett. 107, 264101 (2011).

[81] A. G. Vladimirov, R. Lefever, and M. Tlidi, Phys. Rev. A 84, 043848 (2011).

[82] K. A. Gorshkov and L. A. Ostrovsky, Physica D 3, 428 (1981).

[83] I. S. Aranson, K. A. Gorshkov, A. S. Lomov, and M. I. Rabinovich, Physica D 43, 435 (1990).

[84] Boris A. Malomed, Phys. Rev. A 44, 6954 (1991).

[85] A. G. Vladimirov, J. M. McSloy, D. V. Skryabin, and W. J. Firth, Phys. Rev. E 65, 046606 (2002).

[86] M. Tlidi, A. G. Vladimirov, and P. Mandel, IEEE J. Quantum Electron. 39, 216 (2003).

[87] M. Tlidi, R. Lefever, and A. Vladimirov, in Dissipative Solitons: From Optics to Biology and Medicine, edited by N. Akhmediev and A. Ankiewicz, Lecture Notes in Physics Vol. 751 (Springer, Berlin, 2008), p. 381. 